\title{
Evolution of sexual systems, dispersal strategies and habitat selection in the liverwort genus Radula
}

\author{
Nicolas Devos ${ }^{1,4}$, Matt A. M. Renner ${ }^{2}$, Robbert Gradstein ${ }^{3}$, A. Jonathan Shaw ${ }^{4}$, Benjamin Laenen ${ }^{1}$ and \\ Alain Vanderpoorten ${ }^{1}$ \\ ${ }^{1}$ Institut de Botanique, Université de Liege, B-22 Sart Tilman, B-4000 Liege, Belgium; ${ }^{2}$ National Herbarium of New South Wales, Royal Botanic Gardens \\ Sydney, Mrs Macquaries Road, Sydney, NSW 2000, Australia; ${ }^{3}$ Dept. Systématique et Evolution, Muséum National d'Histoire Naturelle, 57 rue Cuvier, \\ 75231 Paris cedex 05, France; ${ }^{4}$ Biology Department, Duke University, Box 90338 Durham, NC 27708, USA
}

Author for correspondence:

Nicolas Devos

Tel: +19196607298

Email:nd28@duke.edu

Received: 7 March 2011

Accepted: 26 April 2011

New Phytologist (2011) 192: 225-236

doi: 10.1111/j.1469-8137.2011.03783.x

Key words: bryophyte, comparative methods, epiphytism, life-history theory, liverwort, Radula, sexual system, trade-off.

\section{Summary}

- Shifts in sexual systems are among the most common and important transitions in plants and are correlated with a suite of life-history traits. The evolution of sexual systems and their relationships to gametophyte size, sexual and asexual reproduction, and epiphytism are examined here in the liverwort genus Radula.

- The sequence of trait acquisition and the phylogenetic correlations between those traits was investigated using comparative methods.

- Shifts in sexual systems recurrently occurred from dioecy to monoecy within facultative epiphyte lineages. Production of specialized asexual gemmae was correlated to neither dioecy nor strict epiphytism.

- The significant correlations among life-history traits related to sexual systems and habitat conditions suggest the existence of evolutionary trade-offs. Obligate epiphytes do not produce gemmae more frequently than facultative epiphytes and disperse by whole gametophyte fragments, presumably to avoid the sensitive protonemal stage in a habitat prone to rapid changes in moisture availability. As dispersal ranges correlate with diaspore size, this reinforces the notion that epiphytes experience strong dispersal limitations. Our results thus provide the evolutionary complement to metapopulation, metacommunity and experimental studies demonstrating trade-offs between dispersal distance, establishment ability, and life-history strategy, which may be central to the evolution of reproductive strategies in bryophytes.

\section{Introduction}

For more than two centuries, the diversity of plant sexual systems has captured the curiosity and imagination of biologists and stimulated fruitful research by both ecologists and population geneticists (Cheptou \& Schoen, 2007). In angiosperms, only $6 \%$ of species are dioecious, but their scattered taxonomic positions within the group suggest that shifts in sexual systems, mostly towards dioecy from monoecious ancestors, occurred independently multiple times (Renner \& Ricklefs, 1995). The evolutionary transition from sexual monomorphism to dimorphism is governed by three factors, which are the optimal allocation of resources to female and male function, the genetic control of sex expression, and the fitness consequences of selfing and outcrossing (Barrett, 2002).
Dioecious species may benefit from reduced inbreeding depression, but, as only females produce seeds, dioecious females must produce more or better seeds than their hermaphrodite or monecious counterparts and/or disperse those seeds to an equivalent number of suitable sites to ensure an equivalent number of progeny (Charlesworth \& Charlesworth, 1978). In fact, theoretical work suggests that, in order for dioecy to persist, dioecious individuals need a substantial fitness and/or dispersal advantage over cosexuals, even when the number of seeds per female is twice that of cosexuals (Heilbuth et al., 2001).

In bryophytes, where sexual reproduction depends on sperm swimming to eggs via free water, separation of sexes can present serious problems for successful sexual fusion and dioecious species therefore often fail to reproduce sexually (Vanderpoorten \& Goffinet, 2009). In a study on the 
reproductive biology of British mosses, Longton (1997) found that $87 \%$ of the species for which sporophytes are unknown are dioecious, whereas sporophytes are commonly found in $83 \%$ of the monoecious species. Despite this, $c$. $70 \%$ of liverworts and $60 \%$ of mosses are dioecious (Vanderpoorten \& Goffinet, 2009). Along with the frequent independent evolution of dioecy among angiosperm lineages and other major groups of land plants (Renner \& Ricklefs, 1995; Sakai et al., 1995), this suggests that separation of sexes is not necessarily associated with increased extinction risks. In fact, the uncertainty of sexual reproduction in dioecious bryophyte species is thought to have selected for a series of life-history traits that promote dispersal (Wilson \& Harder, 2003; During, 2007). In particular, a strong association between dioecy and the ability to produce specialized asexual propagules has long been known to exist (Longton \& Schuster, 1983; Hedderson \& Longton, 1995; During, 2007). Crawford et al. (2009), however, failed to find support for a phylogenetic correlation between the production of specialized propagules and separate sexes in a recent comparative study across mosses. That study revealed that organisms with separate sexes tend to be significantly larger than monoecious ones and characterized by strong, competitive vegetative growth. This correlation, also observed in angiosperms (de Jong, 2000), is consistent with the hypothesis that dioecious taxa compensate for the low production of spores or seeds by increased size and life expectancy.

These trade-offs between sexual systems and other lifehistory traits have led to the concept of life-history strategies, which can be defined as a recurrent combination of life-history traits that are predicted to occur in response to particular ecological conditions (During, 1992). For example, given the presumed ephemeral nature of their substrates, and the importance of dispersal and colonization (Snäll et al., 2005), epiphytes are typically hermaphroditic (van Dulmen, 2001) and expected to undergo substantial inbreeding to ensure reproductive success (Hooper \& Haufler, 1997; Chiou et al., 2002). In fact, a survey of 15 vascular montane cloud forest epiphytes in Costa Rica showed that all were self-compatible, which represents 'the highest proportion of self-compatible species documented within any life form at any tropical site' (Bush \& Beach, 1995).

A number of studies have employed phylogenies to investigate evolutionary transitions in plant sexual systems (see Case et al., 2008 for a review). The aims of those studies were primarily to estimate the number of times particular traits arose, to identify their life-history trait correlates, and to determine the local direction of changes. In practice, however, mapping traits onto trees is not necessarily straightforward, particularly for those with a high degree of lability that erodes phylogenetic signal (Weller \& Sakai, 1999). Maximum parsimony has been the most widely used principle for inferring character-state transformations from a phylogenetic tree (Smith, 2010). Under the parsimony criterion, which implicitly involves transition rates being equal among states, the solution requiring the minimum amount of changes on a tree is singled out, regardless of branch lengths. A range of alternative reconstructions on the same tree, however, exists, creating a source of error known as 'within-tree uncertainty' (Pagel et al., 2004). Model-based methods, by contrast, directly use branch lengths to determine the probability of change along a branch, allow transition rates between states to vary, and enable an assessment of the accuracy of reconstructions. Because they implement actual transition rates, modelbased methods further enable one to make and test hypotheses regarding evolutionary processes (Pagel, 1994). Looking across multiple shifts, it is, in particular, possible to test for significant correlations among traits and determine the contingency and order of acquisition of those traits independently from the ancestral state reconstructions themselves (Pagel, 1994). Most recently, Markov chain Monte Carlo methods have offered a formal framework to sample model parameters and phylogenies according to their posterior probabilities, thereby taking both within-tree and among-tree uncertainty into account and increasing the statistical power as compared with single-tree approaches (Pagel \& Lutzoni, 2002).

In the present study, we take advantage of the leafy liverwort genus Radula to investigate the evolution of sexual systems and explore trade-offs in life-history traits involved in those transitions. The genus includes c. 200 species (Yamada, 2003) and has an almost world-wide distribution, with the majority of the species occurring in humid, tropical or warm-temperate regions. The species usually grow as epiphytes on bark or living leaves, and less commonly on rock, in a variety of habitats varying from rainforest to alpine tundra, and from sea level to over $4000 \mathrm{~m}$ in elevation. Phylogenetically, Radula has a rather isolated position within the leafy liverworts and is classified in its own family and order (Crandall-Stotler et al., 2009). Morphologically, the genus stands out by virtue of its unique, Radula-type branching system, rhizoids being produced on leaf lobules rather than on stems, the total absence of underleaves, the possession of very large, often single, brown oil bodies in leaf cells, and the development of a tubular or trumpet-like, dorsiventrally flattened perianth which surrounds and protects the developing sporophyte. The genus exhibits a wide range of reproductive characteristics in terms of sexual condition and production of asexual propagules. The genus has both obligate and facultative epiphytes and therefore allows the unique opportunity to investigate possible correlations between this particular trait and sexual systems.

Using a molecular species-level phylogeny of the genus, we studied the evolution of its sexual systems in relation to four life-history traits (reproductive effort, asexual repro- 
duction, gametophyte size and facultative vs obligate epiphytism), implementing competing explicit models of trait evolution (Barker et al., 2007). We then tested phylogenetic correlations among those traits and addressed the following questions. What is the ancestral sexual system in Radula? Are shifts from one sexual system to another random or directional, thereby suggesting their adaptive potential? To what extent are shifts in sexual system correlated with shifts from a facultative to an obligate epiphytic condition; that is, is monoecy favoured in strictly epiphytic lineages in response to the necessity to disperse in a dynamic environment? Do dioecious species compensate for the reduced levels of sporophyte production by increasing life-span and the production of asexual gemmae?

\section{Materials and Methods}

\section{Taxon sampling, DNA extraction, PCR, and sequencing}

Ninety-three of the $c .200$ species of Radula were sampled, depending on the availability of suitable material for DNA studies (see Supporting Information, Table S1 for voucher information). Our sampling covered the entire distribution range of the genus, with samples from Asia, Australia, New Zealand, Africa, Europe, and North and South America as well as various islands and archipelagos such as Reunion, Madagascar, Madeira, the Azores and the Canary Islands. It also included representatives from the four subgenera and major sections recognized within Radula. Frullania moniliata, Jubula pennsylvanica and Porella pinnata were employed as outgroups based upon the results of a genuslevel molecular phylogenies of liverworts (Heinrichs et al., 2005; Forrest et al., 2006).

Total genomic DNA was isolated using a Cetyltrimethylammonium Bromide (CTAB) extraction protocol (Doyle \& Doyle, 1987) without RNase treatment and purified using the Geneclean ${ }^{\circledR}$ III Kit (MP Biomedicals, Solon, $\mathrm{OH}, \mathrm{USA})$. The chloroplast atpB-rbcL, $p s b T-p s b H, p s b A-$ $\operatorname{trn} H, r p s 4, \operatorname{trn} G$ and $\operatorname{trn} L-F$ DNA regions were amplified and sequenced for all accessions (see Table S1 for GenBank accession numbers). Sequences for the outgroup taxa were downloaded from GenBank and added to the sequences generated for this study. The primers used for PCR amplification of those six chloroplast regions are listed in Table 1. PCR products were purified before sequencing using the Exonuclease enzymatic reaction (New England Biolabs, Ipswich, MA, USA). Forward and reverse sequencing of the purified amplicons was conducted using the $\mathrm{BigDye}{ }^{\circledR}$ Terminator v. 3.1 cycle sequencing kit (Applied Biosystems, Foster City, CA, USA).

Forward and reverse sequences were assembled and edited using Sequencher 4.01 (Gene Codes Corporation, 1998). Contigs were aligned using Muscle 3.6 (Robert, 2004) and the alignments obtained were manually refined using MacClade 4.1 (Maddison \& Maddison, 1992).

\section{Phylogenetic analyses}

The nucleotide data matrix, available from TreeBASE at http://www.treebase.org under study number S11286, was submitted to a Bayesian phylogenetic inference using MrBayes 3.1 (Ronquist \& Huelsenbeck, 2003). The six individual data sets were first analysed separately in order to identify possible incongruence between them. The best-fitting substitution model for each individual locus was selected with the Akaike information criterion using MrModeltest (Nylander, 2004) in conjunction with PAUP* (Swofford, 2002). A General Time-Reversible (GTR) model with gamma-distributed rate variation across sites and a proportion of invariable sites was selected for $p s b A$ $\operatorname{trn} H, \quad r p s 4, \operatorname{trnL}-F, a \operatorname{tp} B-r b c L$, and $\operatorname{trn} G$, while an Hasegawa, Kishino and Yano (HKY) model with gammadistributed rate variation across sites and a proportion of invariable sites was selected for $p s b T-p s b H$. These models were implemented using four independent Markov chain Monte Carlo (MCMC) simulations run for 20000000

Table 1 Primers used in this study for the amplification and sequencing of six chloroplast DNA regions in the leafy liverwort genus Radula

\begin{tabular}{lllll}
\hline Region & Primer & Sequence 5'-3' $^{\prime}$ & Direction & References \\
\hline trnL-trnF & trnC & CGAAATCGGTAGACGCTACG & Forward & Taberlet et al. (1991) \\
& trnF & ATTTGAACTGGTGACACGAG & Reverse & Taberlet et al. (1991) \\
trnG & trnGF & ACCCGCATCGTTAGCTTG & Forward & Pacak \& Szweykowska-Kulinska (2000) \\
& trnGR & GCGGGTATAGTTTAGTGG & Reverse & Pacak \& Szweykowska-Kulinska (2000) \\
rps4 & rps5 & ATGTCCCGTTATCGAGGACCT & Forward & Nadot et al. (1994) \\
& trnas & TACCGAGGGTTCGAATC & Reverse & Souza-Chies et al. (1997) \\
psbA-trnH & trnK2F & GACGAGTTCCGGGTTCGA & Forward & C. J. Cox, unpublished \\
& trnHR & GAACGACGGGAATTGAAC & Reverse & C. J. Cox, unpublished \\
psbT-psbH & psbT & ATGGAAGCWTTAGTWTATACWTT & Forward & Krellwitz et al. (2001) \\
& psbH & GTHCCCCARCCDGGDRVHACTTTWCC & Reverse & Krellwitz et al. (2001) \\
atpB-rbcL & atpb672F & TTGATACGGGAGCYCCTCTWAGTGT & Forward & P. G. Wolf, unpublished \\
& atpb910R & TTCCTGYARAGANCCCATTTCTGT & Reverse & P. G. Wolf, unpublished \\
\hline
\end{tabular}


generations and sampled every 10 000th generation. Burnin length and convergence between the four runs were confirmed by comparing the trace files of each run using TRACER 1.4 (Rambaut \& Drummond, 2007). After discarding the burn-in, outputs from the four runs were combined for final inference of posterior probabilities of both trees and model parameters.

No taxon was resolved as part of two distinct clades supported each by $>70 \%$ posterior probabilities in analyses of the individual data sets. These partitions were therefore combined into a single matrix that was submitted to a heterogeneous Bayesian analysis using the settings and implementing, for each partition, the best-fitting substitution model detailed in the previous paragraph. Model parameters (with the exception of branch lengths and topology) were unlinked using the 'unlink' command in MRBAYES such that each partition had its own set of parameters.

\section{Evolution and correlated evolution in life-history traits}

The sexual system (dioecious (0) or monoecious (1)) of each species was scored from the literature (See Table S2 for a list of the references consulted). Nine monoecious species were sampled, and this reflected the $10 \%$ rate of monoecy (16 species) within the genus (S. R. Gradstein, unpublished observations). In order to determine the correlation between sexual systems, sexual and asexual reproduction, habitat conditions, and life expectancy, the following four traits were also scored from the same sources: presence (1) or absence (0) of specialized asexual propagules (gemmae or caducous leaf lobes); obligate (1) or facultative epiphytes occurring on a wide range of substrates (0); gametophyte size: shoot width $<2.0 \mathrm{~mm}(0)$ or $>2.0 \mathrm{~mm}$ (1); sexual reproduction: perianth never or very rarely produced $(0)$ or occasionally to frequently present (1). The absence of perianth formation was used here as a proxy for the absence of sporophytes and, as a consequence, of sexual reproduction. With the exceptions of Plagiochila and Gymnocolea, perianths in liverworts only develop when sporophytes are initiated after sexual reproduction (Schuster, 1966; Vanderpoorten \& Goffinet, 2009). Obligate vs facultative epiphytism was similarly taken as a proxy for habitat stability. In fact, all Radula species are restricted to hard surfaces, basically living bark (or leaves) or rocks (Gradstein et al., 2001). Given the longer duration of the latter habitat (Söderström \& Herben, 1997) coupled with the high turnover rate of cryptogamic communities on a tree (Barkman, 1958), this means that an individual of a facultative epiphytic Radula species has theoretically a longer life expectancy than an individual of an obligate one.

Other potentially important life-history traits, such as spore size and seta length, are sometimes implicated in trade-offs with sexual system. Those life-history traits were, however, not included in this study. In fact, only a small percentage of species have been observed with sporophytes, which hinders the scoring of those sporophytic characters and would ultimately introduce too many missing data in the data set.

For each investigated trait, ancestral states were reconstructed on the trees generated by MRBAYES after pruning the outgroups. The probabilities of change on a branch were calculated by estimating the instantaneous forward $\left(q_{01}\right)$ and backward $\left(q_{10}\right)$ rates between the two states by implementing the 'Multistate' Markov model (Pagel et al., 2004) in BayesTraits 1.0 (available from http://www.evolution.rdg.ac.uk). An MCMC was used to visit, at each iteration, the space of rate parameter values and sample one of the trees generated by the MrBAYES analysis. The likelihood of the new combination was calculated and this new state of the chain was accepted or rejected following evaluation by the Metropolis-Hastings term. The rate at which parameters were changed ('ratedev') was set at the beginning of each run so that the acceptance rate of the proposed change globally ranged between 20 and 40\%. In the absence of information on rates, uniform distributions ranging from 0 to 100 were used as priors. The chain was run for 50000000 generations and sampled for rate parameters and state probabilities at the nodes of interest every 10000 generations. In order to circumvent the issue associated with the fact that not all the trees necessarily contain the internal nodes of interest, reconstructions were performed using a 'most recent common ancestor' approach (Pagel et al., 2004).

For each trait, we compared the performances of six competing explicit evolutionary models to describe trait evolution, as advocated by Barker et al. (2007) for traits with a weak phylogenetic signal (see also Vanderpoorten et al., 2010 for a review). The first two models were the unconstrained two-rate $\left(q_{01} \neq q_{10}\right)$ and single-rate $\left(q_{01}=q_{10}\right)$ models, respectively, wherein $q_{01}$ and $q_{10}$ are sampled from a uniform distribution with a $0-100$ range. In the third model, the forward rate $q_{01}$ was set to 0 . This model, which corresponds to the 'ML-root' model of Barker \& Pagel (2005) and Barker et al. (2007), is consistent with a scenario in which the state is already present at the root and subsequently lost in different lineages, without any possibilities of being gained again. The fourth model was used to describe a situation similar to the previous one, but low values of forward transition rates allow for rare gain events to happen. This situation was described by a model in which $q_{01}$ was forced to be very low (i.e. was sampled from a uniform distribution with a range of $0-1$ ), whereas no constraint was imposed on $q_{10}$ (which was sampled from a uniform distribution with a range of $0-100)$. The fifth and sixth models are the equivalent of models 3 and 4 , but with $q_{10}$ either constrained to be equal to 0 or sampled from a $(0-1)$ uniform prior distribution. The two last models are consistent with a 
scenario in which the state, once gained, can never be lost again (model 5) or has a very small probability of being lost (model 6).

Bayes factors (BFs) were used to determine which model(s) best fitted the data, and the best-fitting model was subsequently used for trait mapping. In order to contrast alternative hypotheses regarding the ancestral state at key nodes of the phylogeny, we used the 'local' approach, wherein the significance of the reconstruction is explicitly tested at each node of interest (Pagel, 1999). For that purpose, we successively fixed each of the latter at one of the two states it can take ( 0 or 1$)$. Then, an MCMC was used to visit the sample of trees generated by the MrBAYES analysis and the space of rate parameter values. A second, independent chain was run to sample rate parameters and derive overall likelihoods of the reconstructions when the node was fixed at its alternative state. BFs were then used to determine the support for alternative states.

In order to investigate correlated evolution among traits, each pair of traits was analysed successively using two alternative models: a four-rate model describing independent evolution between traits, and an eight-rate model describing correlated evolution (Pagel, 1994) (Fig. 1a,b). Both models were implemented in a Bayesian context and BFs were used to determine whether the dependent or independent model provided a significantly better description of trait evolution. Contingency tests were then performed to detect whether evolution of trait A (sexual system in Fig. 1b) depends upon the state in trait B (epiphytism in Fig. 1b), or vice versa. For example, $q_{13}>q_{24}$ indicates that a shift in the sexual system from 0 to 1 is more likely when epiphytism is at state 0 , whereas $q_{12}>$ $q_{43}$ indicates that a shift in epiphytism from state 0 to 1 is more likely when the sexual condition is at state 0 (Fig. 1b). Contingency tests thus involve restricting one rate to be equal to the other, re-running the analysis, and comparing the difference of fit with the unconstrained analysis using BFs.

\section{Results}

\section{Ancestral state reconstructions}

Six thousand trees, whose $50 \%$ majority-rule consensus is presented in Fig. 2, were sampled from the MRBAYES inference and used for trait mapping and comparative analyses. The unconstrained two-rate model (model 1) was systematically selected amongst the six competing models of trait evolution (Table 2) as the best-fitting model and was thus used for ancestral state reconstructions. Local ancestral state reconstructions of the sexual condition, sexual reproduction, and the presence or absence of asexual propagules (Fig. 3) showed that all shifts occurred from dioecy to monoecy; from presence to absence of sporophytes; and from absence to presence of asexual propagules, respectively. Shifts in the sexual condition and the presence or absence of asexual propagules were recurrent and occurred at very shallow nodes of the phylogeny.

At least six species of Radula evolved from being able to grow on a range of substrates to being obligate epiphytes growing on leaves and/or bark (Fig. 3; see Radula recubans, Radula reflexa, Radula fendleri, Radula quadrata, Radula physoloba and Radula gottscheana). One reversal from obligate to facultative epiphyte was reconstructed (Fig. 3; see Radula pulchella).

\section{Correlations among traits}

Pairwise trait comparisons revealed that the model of independent evolution was favoured over the model of correlated evolution based upon the BFs for five pairs of traits, namely sexual system and production of asexual propagules;

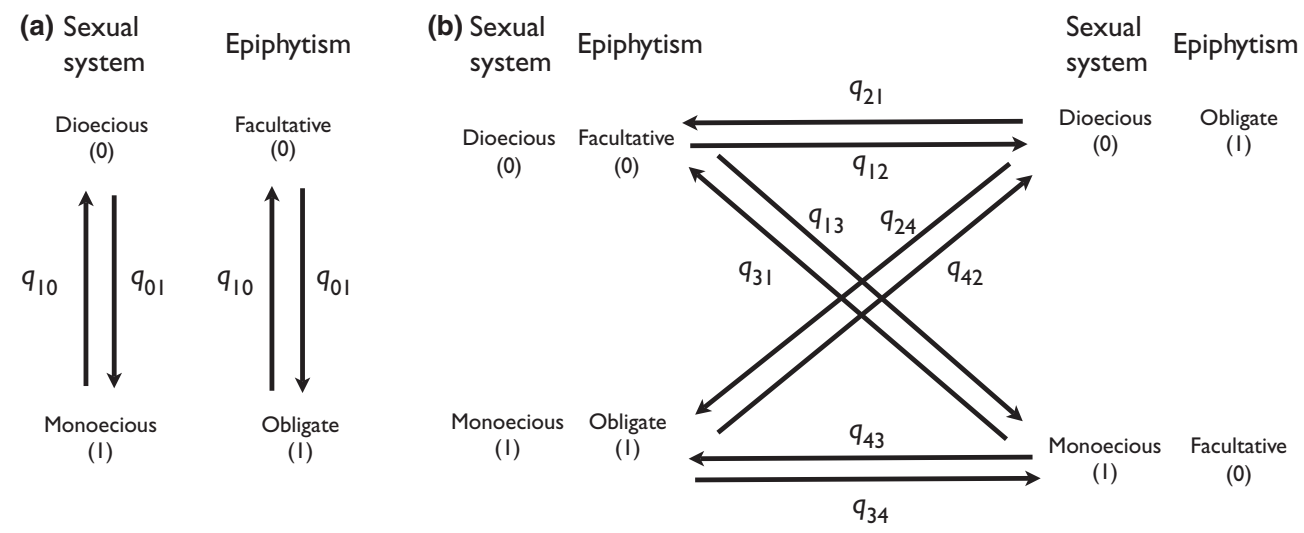

Fig. 1 Models of trait evolution for two binary traits, sexual system and epiphytism. (a) Traits evolve independently from each other. The evolution of each trait is described by a forward rate $q_{01}$ for shifts from state 0 to state 1 , and a backward rate $q_{10}$ for the reverse shift. (b) Traits evolve in a correlated fashion, such that the rate of change in one trait depends upon the background state of the other. Dual transition is not allowed. Joint evolution of the two traits is thus described by an eight-rate model. 


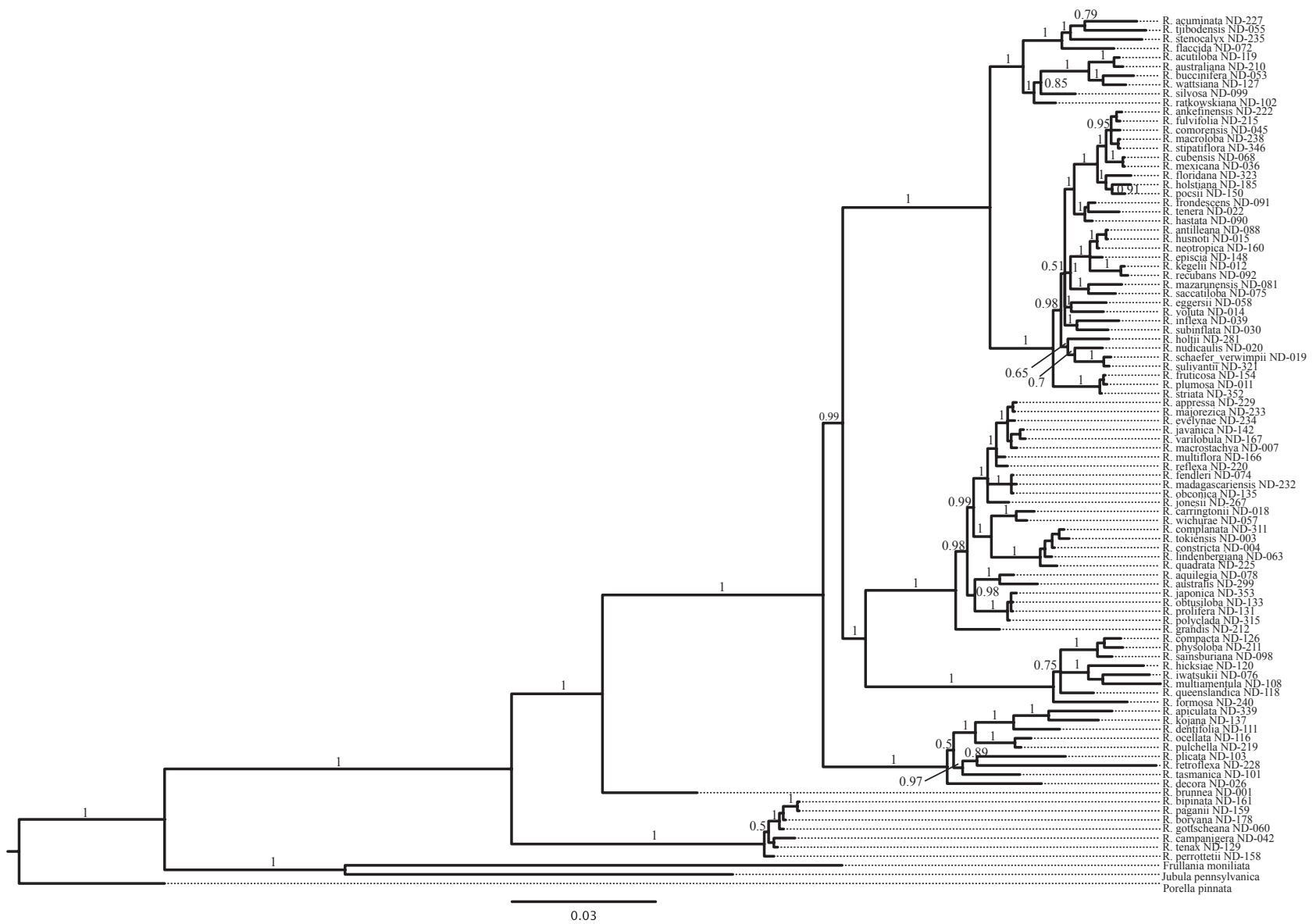

Fig. 2 Fifty per cent majority-rule consensus of the trees sampled by a Bayesian analysis of 93 species of Radula as implemented in MRBAYES 3.1. Branch lengths were averaged over the 6000 trees obtained after removing the burn-in. Numbers above branches correspond to their posterior probability.

Table 2 Harmonic mean of the log-likelihoods associated with the reconstruction of trait evolution for six competing explicit evolutionary models (see text for details) in the liverwort genus Radula

\begin{tabular}{|c|c|c|c|c|c|c|c|c|}
\hline \multirow[b]{2}{*}{ Trait } & \multicolumn{2}{|l|}{ States } & \multicolumn{6}{|l|}{ Models } \\
\hline & 0 & 1 & $2 q$ unconstrained & $1 q$ & $q_{01}=0$ & $\begin{array}{l}q_{01}=(0-1), q_{10} \\
\text { unconstrained }\end{array}$ & $q_{10}=0$ & $\begin{array}{l}q_{10}=(0-1), q_{01} \\
\text { unconstrained }\end{array}$ \\
\hline Sexual system & Dioecious & Monoecious & 35.79 & 39.92 & & 51.27 & 49.61 & 36.52 \\
\hline Asexual propagules & Absent & Present & 57.81 & 59.28 & 76.23 & 74.31 & 77.09 & 70.66 \\
\hline Epiphytism & Facultative & Obligate & 60.48 & 59.82 & 94.47 & 88.58 & 72.81 & 72.91 \\
\hline Gametophyte size & $<2.0 \mathrm{~mm}$ & $>2.0 \mathrm{~mm}$ & 66.35 & 65.68 & 87.40 & 86.41 & 98.95 & 96.40 \\
\hline Sexual reproduction & Absent & Present & 45.42 & 49.29 & 54.49 & 54.94 & 72.64 & 66.92 \\
\hline
\end{tabular}

The best-fitting models, as determined by the Bayes factors, are in bold.

sexual system and sexual reproduction; gametophyte size and production of asexual propagules; gametophyte size and epiphytism; and epiphytism and sexual reproduction. Three pairs of traits were significantly correlated: sexual system and epiphytism; production of gemmae and epiphytism; and production of gemmae and failure to reproduce sexually. The results of contingency tests suggest that sexual system did not influence the evolution of epi- phytism but rather depended upon it (Table 3). Transitions to monoecy were more likely within facultative epiphyte lineages than obligate ones, as indicated by a $q_{13}$ rate of 32.22 compared with a $q_{24}$ rate of 6.18 , (Table 3 ).

Production of asexual propagules seemed to be favoured within facultative epiphytic lineages, as indicated by a $q_{13}$ rate of 60.37 compared with a $q_{24}$ rate of 14.23 (Table 3). A significant correlation was also found between the 
New

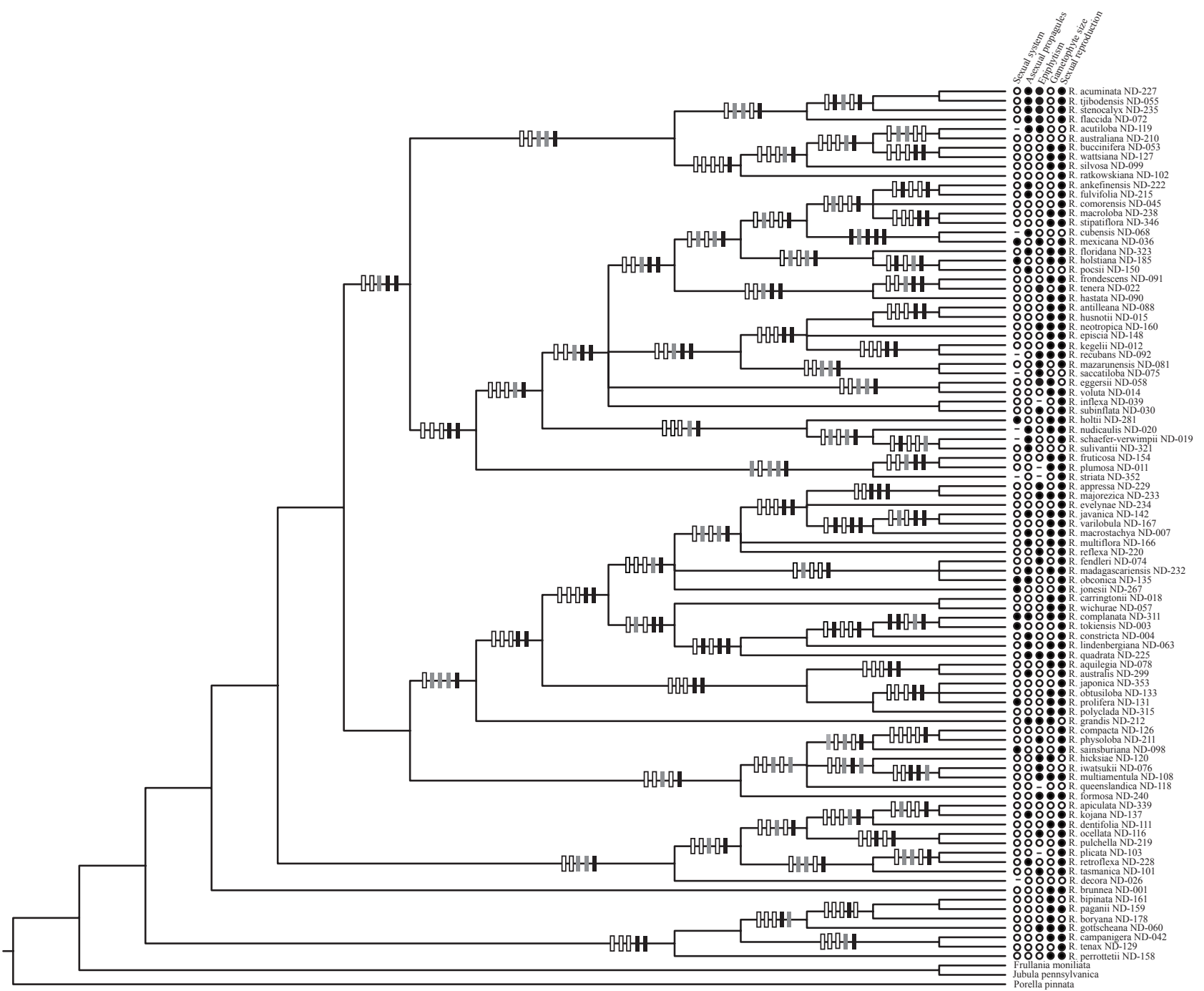

Fig. 3 Cladogram of the $50 \%$ majority-rule consensus of the trees (burn-in removed) sampled by a Bayesian analysis of 93 species of Radula as implemented in MRBAYES 3.1. States for each terminal node are given for the following traits: sexual system (dioecious (0/white) or monoecious ( $1 /$ black)). Presence (1/black) or absence (0/white) of asexual propagules. Obligate ( $1 /$ black) or facultative epiphytes ( $0 /$ white). Gametophyte size: shoot width $<2.0 \mathrm{~mm}(0 /$ white $)$ or $>2.0 \mathrm{~mm}(1 /$ black). Sexual reproduction: perianth never or very rarely produced ( $0 /$ white) or occasionally to frequently present (1/black). Ancestral states at internal nodes for each of those traits are represented by a bar along the branch leading to that node. Those bars are in the same order as the states given for terminal nodes. White and black bars indicate significant support for states 0 and 1, respectively, while grey bars indicate that the reconstruction was ambiguous.

production of asexual diaspores and failure to reproduce sexually, but the associated contingent test was not significant $(\mathrm{BF}=1.22$; Table 3). The two other phylogenetic correlations (sexual system vs gametophyte size and gametophyte size vs sexual reproduction) were not significant (Table 3).

\section{Discussion}

\section{Evolution of sexual systems}

Two lines of evidence suggest that evolution of sexual systems in Radula has been directional. First, the model corresponding to a scenario in which monoecy, once gained, has a very small probability of being lost fits the data as well as the unconstrained model. Secondly, reconstructions of sexual system evolution clearly indicate that all six inferred transitions were from dioecy to monoecy, and never the reverse between nodes with significant state assignment. In bryophytes, the monoecious transition has traditionally been viewed as a result of essentially random polyploidization events (see Crawford et al., 2009 for a review). A comparative analysis across mosses in a phylogenetic framework recently failed, however, to demonstrate a significant correlation between transitions from nonpolyploid gametophytes with separated sexes to polyploid 
Table 3 Harmonic mean $L$ of competing models (independent evolution I, correlated evolution $D$, and contingency test) describing the evolution of pairs of characters in the liverwort genus Radula and associated Bayes factors (BFs)

\begin{tabular}{|c|c|c|c|c|}
\hline Hypothesis testing & Test & \multicolumn{2}{|c|}{ Harmonic mean of $L$ of competing models } & $\mathrm{BF}$ \\
\hline \multicolumn{5}{|l|}{ Sexual system and epiphytism } \\
\hline Correlated evolution & $L(\mathrm{I})<L(\mathrm{D})$ & $L(I)=96.47$ & $L(D)=94.94$ & 3.06 \\
\hline Sexual system depends upon epiphytism & $q_{13} \neq q_{24}$ & $\begin{array}{l}L(\mathrm{D})=94.94 \\
q_{13}=32.22 \pm 12.04 \\
q_{24}=6.18 \pm 6.10\end{array}$ & $L\left\{(\mathrm{D}) \mid q_{13}=q_{24}\right\}=96.52$ & 3.16 \\
\hline Sexual system and production of asexual propagules & & & & \\
\hline $\begin{array}{l}\text { Correlated evolution } \\
\text { Sexual system and gametophyte size }\end{array}$ & $L(\mathrm{I})<L(\mathrm{D})$ & $L(I)=93.45$ & $L(D)=94.84$ & -2.78 \\
\hline $\begin{array}{l}\text { Correlated evolution } \\
\text { Sexual system and sexual reproduction }\end{array}$ & $L(\mathrm{I})<L(\mathrm{D})$ & $L(I)=104.05$ & $L(D)=104.26$ & 0.02 \\
\hline $\begin{array}{l}\text { Correlated evolution } \\
\text { Gametophyte size and sexual reproduction }\end{array}$ & $L(\mathrm{I})<L(\mathrm{D})$ & $L(I)=80.18$ & $L(D)=81.73$ & 3.1 \\
\hline $\begin{array}{l}\text { Correlated evolution } \\
\text { Gametophyte size and production of asexual propagules }\end{array}$ & $L(\mathrm{I})<L(\mathrm{D})$ & $L(I)=112.16$ & $L(D)=111.86$ & 0.60 \\
\hline $\begin{array}{l}\text { Correlated evolution } \\
\text { Gametophyte size and epiphytism }\end{array}$ & $L(\mathrm{I})<L(\mathrm{D})$ & $L(I)=123.29$ & $L(D)=124.65$ & -2.72 \\
\hline $\begin{array}{l}\text { Correlated evolution } \\
\text { Production of asexual propagules and epiphytism }\end{array}$ & $L(\mathrm{I})<L(\mathrm{D})$ & $L(I)=126.636$ & $L(D)=128.185$ & 3.098 \\
\hline Correlated evolution & $L(\mathrm{I})<L(\mathrm{D})$ & $L(I)=117.12$ & $L(D)=115.13$ & 3.98 \\
\hline Production of asexual propagules depends upon epiphytism & $q_{13} \neq q_{24}$ & $\begin{array}{l}L(D)=115.13 \\
q_{13}=60.37 \pm 18.57 \\
q_{24}=14.23 \pm 14.57\end{array}$ & $L\left\{(\mathrm{D}) \mid q_{13}=q_{24}\right\}=116.39$ & 2.52 \\
\hline Production of asexual propagules and sexual reproduction & & & & \\
\hline Correlated evolution & $L(\mathrm{I})<L(\mathrm{D})$ & $L(I)=105.55$ & $L(D)=103.23$ & 4.64 \\
\hline $\begin{array}{l}\text { Production of asexual propagules depends upon } \\
\text { sexual reproduction }\end{array}$ & $q_{13} \neq q_{24}$ & $\begin{array}{l}L(D)=103.23 \\
q_{13}=47.45 \pm 25.50 \\
q_{24}=48.62 \pm 15.86\end{array}$ & $L\left\{(\mathrm{D}) \mid q_{13}=q_{24}\right\}=102.63$ & -1.20 \\
\hline $\begin{array}{l}\text { Epiphytism and sexual reproduction } \\
\text { Correlated evolution }\end{array}$ & $L(\mathrm{I})<L(\mathrm{D})$ & $L(I)=105.28$ & $L(D)=107.37$ & 4.18 \\
\hline
\end{tabular}

The best-fitting models, as determined by Bayes factors, are in bold. See text for details.

cosexuals (Crawford et al., 2009). The assumption that monoecy is associated with polyploidy is further weakened as polyploidy is extremely rare in liverworts in general (Fritsch, 1991) and in Radula in particular, as indicated by recent chromosome number counts (Zheng \& Zhu, 2009).

In Radula, monoecy appears at the shallowest nodes of the phylogeny, as if it were a recent product of evolution, and its recurrent acquisition (nine shifts from dioecious to monoecious) suggests that it confers a selective advantage. This is consistent with the observation that monoecious bryophyte species tend to display, on average, broader distribution ranges than dioecious ones (see Longton \& Schuster, 1983 for a review). In bryophytes, the advantages of monoecy are twofold. First, monoecy greatly enhances the chances of fertilization (Longton, 1997). This definitely represents a strong selective pressure on the evolution of sexual systems because, owing to the necessity of a film of water between the male and female gametangia, fertilization ranges are extremely limited, which is critical for dioecious species. In the moss Hylocomium splendens, Rydgren et al. (2006) found, for example, that $85 \%$ of the female shoots with sporophytes were situated within a distance of $5 \mathrm{~cm}$ from the nearest male and the greatest distance was
$11.6 \mathrm{~cm}$. Such a constraint is, by contrast, virtually lacking in monoecious species because the distance between sexes is minimal. Secondly, monoecious species have lower sporophytic inbreeding depression than dioecious ones (Taylor et al., 2007). While dioecious species either outcross or undergo intergametophytic selfing (mating among haploid siblings from the same sporophyte), monoecious species are capable of intragametophytic selfing (merging of gametes produced by the same genetic individual), which results in a fully homozygous sporophyte generation. Monoecious species can thus rapidly purge recessive deleterious mutations through intragametophytic selfing while sporophytic inbreeding depression is assumed to occur in dioecious species (Eppley et al., 2007). Intragametophytic selfing inevitably leads to genetic uniformity (McDaniel et al., 2010), but low levels of genetic diversity do not necessarily hamper evolutionary success in the short term (see Karlin et al., 2011 for a review). Maintenance of selected genotypes through selfing might even prove adaptive in specialists of highly selective environments (niche-width hypothesis; Soulé, 1971; but see Dubois et al., 2003). Low levels of genetic variation are, however, assumed to retard evolutionary potential, so that monoecy has commonly 
been viewed in the literature as an 'evolutionary dead-end' (see Barrett, 2002 for a review), triggering the recurrent evolution of dioecy within unrelated lineages (e.g. Renner \& Won, 2001; Miller \& Venable, 2003; Ronse De Craene \& Wanntorp, 2006; Renner et al., 2007; Case et al., 2008) and other mechanisms such as self-incompatibility. In this regard, the appearance of monoecy at the shallowest nodes of the Radula phylogeny could also be interpreted in terms of a recurrent evolution of monoecy followed by extinctions throughout the evolutionary history of the genus.

\section{Sexual systems, dispersal strategies and habitat selection}

Transitions to monoecy were found to be phylogenetically significantly correlated with epiphytism in Radula. However, comparison of trait acquisition rates suggests that it is not the sexual system that determines the evolution of epiphytism, but the reverse. Transitions to monoecy are, in fact, significantly more likely to occur in facultative epiphytic lineages than in obligate ones. As obligate epiphytes must necessarily disperse from one host to another within a shorter time frame than do facultative epiphytic species occurring on more stable substrates, these observations are, at first sight, counterintuitive. Unstable habitats tend indeed to favour increased reproductive effort and the production of smaller, more numerous offspring, whereas stable habitats are expected to favour increased investment in vegetative growth, reduced reproductive effort, and the production of fewer and larger offspring (Stearns, 1989; Kozlowski, 1992). In fact, Hedderson \& Longton (2008) observed a trade-off between effort expended on spore production and that expended on vegetative proliferation among populations of the moss Polytrichum juniperinum from environments with contrasting habitat stability. Monoecy might not be selected for in epiphytic lineages because gravity makes sperm dispersal distances longer, potentially allowing dioecious bryophytes to reproduce sexually despite the separation in space between male and female individuals (Longton, 1997). In addition, two possible explanations may account for the lower probability of monoecious transition in obligate vs facultative epiphytes in Radula.

First, transition to epiphytism in bryophytes, and mosses in particular, is associated with a suite of gametophytic morphological transformations (see Huttunen et al., 2004 for a review) that probably competes with sporophyte production in terms of resource allocation. Sporophyte production indeed correlates with higher shoot mortality among females (Pohjamo \& Laaka-Lindberg, 2003), reduced size of vegetative offshoots, and reduced branching (Rydgren \& Økland, 2003; Stark et al., 2009). This hypothesis is, however, weakened in Radula because no correlation was observed between gametophyte size and the presence of sexual reproduction, and because no differences in gametophyte morphology are evident between facultative and obligate epiphytes.

Secondly, epiphytes might preferentially disperse by means of asexual diaspores. Production of asexual diaspores is indeed regarded as an adaptation to the spatio-temporal variation in habitat quality and water supply because asexual propagules allow for earlier reproduction and are less sensitive to habitat quality than spores (Löbel, 2009). Asexual reproduction was further shown to result in the production of new gametophytes at a faster rate than sexual reproduction in the moss Syntrichia ruralis (Mishler \& Newton, 1988). In addition, no trade-off between shoot mortality and asexual reproduction has been found to date and, as opposed to previous assumptions (Kimmerer, 1993), vegetative diaspores are also capable of long-distance dispersal (Pohjamo et al., 2006). However, as opposed to the expectations from life-history theory, the contingency test indicated that production of specialized asexual propagules is favoured within facultative epiphytic lineages of Radula.

One interpretation is that entire gametophyte fragments would be equally if not preferentially dispersed by animals in a forest environment with limited wind intensity as compared with spores or specialized gemmae. Bryophyte fragments have indeed been found on roe deer in a forest environment (Heinken et al., 2001). Accidental ingestion may also occur, so that viable fragments could potentially be dispersed via passage through the digestive tract of vertebrates such as flying foxes and birds (Parsons et al., 2007). To date, however, evidence for zoochory in bryophytes is very limited and its impact on dispersal unknown.

An alternative interpretation is that specialized gemmae necessarily germinate into a protonema that will pass through all the successive ontogenetical stages (asexual reproduction sensu Mogie (1992), although not from the embryonic state). Passage through the very sensitive early developmental stages, and especially the protonema, which is more sensitive to drought than both the diaspores and the gametophyte (Proctor et al., 2007), might be critical for epiphytes, which colonize a rather extreme habitat in terms of the temporal range of temperature, physico-chemical and humidity conditions (Leon-Vargas et al., 2006). Therefore, a perhaps more efficient strategy for epiphytic liverworts is to disperse vegetatively by means of whole plants or large gametophytic fragments, which can detach from the substrate, disperse, and resume growth from the apical meristematic cell (asexual propagation) at a new suitable micro-site, thereby avoiding the critical protonemal phase. In fact, establishment rates in epiphytic bryophytes were experimentally shown to be higher from gametophytes than from gemmae or spores (Löbel et al., 2009). By dispersing preferentially through whole gametophyte fragments, obligate epiphytic Radula species could enhance the chances of establishment within a habitat prone to variation in quality 
and water content. In the meantime, as dispersal ranges correlate with diaspore size (Pohjamo et al., 2006), this dramatically decreases their medium and long-distance dispersal ability, reinforcing the notion that epiphytes experience substantial dispersal limitations (Snäll et al., 2004a,b, 2005; Löbel et al., 2006a,b). Our results thus provide the evolutionary complement to previous metapopulation, metacommunity and experimental studies demonstrating trade-offs between dispersal distance, establishment ability, and life-history strategy (Löbel \& Rydin, 2010), which may be central to the evolution of reproductive strategies in bryophytes.

\section{Acknowledgements}

Many thanks are due to three anonymous reviewers for their constructive comments on a previous draft of this paper. A. Schäfer-Verwimp, T. Pocs, and the Helsinki $(\mathrm{H})$, Liege (LG), Missouri (MO), New York (NY), Edinburgh (E), Eszterhazy Karoly College (EGR), Auckland (AK), Goetingen (GOET), and the Australian National Botanical Gardens (CBG) herbaria are gratefully acknowledged for the loan of specimens. N.D., B.L. and A.V. acknowledge financial support from the Belgian Funds for Scientific Research (FNRS) and the Fonds Léopold III. This research was also supported by NSF Grant EF-0531730-002 to A.J.S.

\section{References}

Barker D, Meade A, Pagel M. 2007. Constrained models of evolution lead to improved prediction of functional linkage from correlated gain and loss of genes. Bioinformatics 23: 14-20.

Barker D, Pagel M. 2005. Predicting functional gene links from phylogenetic-statistical analyses of whole genomes. PLoS Computational Biology 1: e3.

Barkman JJ. 1958. Phytosociology and ecology of cryptogamic epiphytes. Assen: van Gorcum.

Barrett SCH. 2002. The evolution of plant sexual diversity. Nature Review Genetics 3: 274-284.

Bush SP, Beach JH. 1995. Breeding systems of epiphytes in a tropical montane wet forest. Selbyana 16: 155-158.

Case AL, Graham SW, Macfarlane TD, Barrett CH. 2008. A phylogenetic study of evolutionary transitions in sexual systems in Australasian Wurmbea (Colchicaceae). International Journal of Plant Science 169: 141-156.

Charlesworth B, Charlesworth D. 1978. A model for the evolution of dioecy and gynodioecy. American Naturalist 112: 975-997.

Cheptou PO, Schoen DJ. 2007. Combining population genetics and demographical approaches in evolutionary studies of plant sexual systems. Oikos 116: 271-279.

Chiou WL, Farrar DL, Ranker TA. 2002. The sexual systems of some epiphytic Polypodiaceae. American Fern Journal 92: 65-79.

Crandall-Stotler BJ, Stotler RE, Long DG. 2009. Phylogeny and classification of the Marchantiophyta. Edinburgh Journal of Botany 66: $155-198$.

Crawford M, Jesson LK, Garnock-Jones PJ. 2009. Correlated evolution of sexual system and life history traits in mosses. Evolution 63: $1129-1142$.
Doyle JJ, Doyle JL. 1987. Preservation of plant samples for DNA restriction endonuclease analysis. Taxon 36: 715-722.

Dubois S, Cheptou PO, Petit C, Meerts P, Poncelet M, Vekemans X, Lefebvre C, Escarre J. 2003. Genetic structure and sexual systems of metallicolous and nonmetallicolous populations of Thlaspi caerulescens. New Phytologist 157: 633-641.

van Dulmen A. 2001. Pollination and phenology of flowers in the canopy of two contrasting rain forest types in Amazonia, Colombia. Plant Ecology 153: 73-85.

During HJ. 1992. Ecological classifications of bryophytes and lichens. In: Bates JW, Farmer AM, eds. Bryophytes and lichens in a changing environment. Oxford: Clarendon Press, 1-31.

During HJ. 2007. Relations between clonal growth, reproduction and breeding system in the bryophytes of Belgium and The Netherlands. Nova Hedwigia Supplement 131: 133-145.

Eppley SM, Taylor PJ, Jesson LK. 2007. Self-fertilization in mosses: a comparison of heterozygote deficiency between species with combined versus separate sexes. Heredity 98: 38-44.

Forrest LL, Davis EC, Long DG, Crandall-Stotler BJ, Clark A, Hollingsworth ML. 2006. Unraveling the evolutionary history of the liverworts (Marchantiophyta): multiple taxa, genomes and analyses. The Bryologist 109: 303-334.

Fritsch R. 1991. Index to bryophytes chromosome counts. Bryophytorum Bibliotheca 40: 1-352.

Gene Codes Corporation. 1998. Sequencher 4.01 reference, advanced, user friendly software tools for DNA sequencing. Madison, WI: Gene Codes Corporation.

Gradstein SR, Churchill SP, Salazar AN. 2001. Guide to the bryophytes of tropical America. Memoirs of the New York Botanic Garden 86: 1-577.

Hedderson TA, Longton RE. 1995. Patterns of life history variation in the Funariales, Polytrichales, and Pottiales. Journal of Bryology 18: 639-675.

Hedderson TA, Longton RE. 2008. Local adaptation in moss life histories: population-level variation and a reciprocal transplant experiment. Journal of Bryology 30: 1-11.

Heilbuth JC, Ilves KL, Otto SP. 2001. The consequences of dioecy for seed dispersal: modeling the seed-shadow handicap. Evolution 55 : 880-888.

Heinken T, Lees R, Raudnitschka D, Runge S. 2001. Epizoochorous dispersal of bryophyte stem fragments by roe deer (Capreolus capreolus) and wild boar (Sus scrofa). Journal of Bryology 23: 293-300.

Heinrichs J, Gradstein SR, Wilson R, Schneider H. 2005. Towards a natural classification of liverworts (Marchantiophyta) based on the chloroplast gene rbcL. Cryptogamie Bryolgie 26: 131-150.

Hooper EA, Haufler CH. 1997. Genetic diversity and bredding system in a group of Neotropical epiphytic ferns (Pleopeltis, Polypodiaceae). American Journal of Botany 84: 1664-1674.

Huttunen S, Ignatov MS, Müller K, Quandt D. 2004. Phylogeny and evolution of epiphytism in the three moss families Meteoriaceae, Brachytheciaceae, and Lembophyllaceae. Monographs in Systematic Botany 98: 328-361.

de Jong TJ. 2000. From pollen dynamics to adaptive dynamics. Plant Species Biology 15: 31-41.

Karlin EF, Andrus RE, Boles SB, Shaw AJ. 2011. One haploid parent contributes $100 \%$ of the gene pool for a widespread species in northwest North America. Molecular Ecology 20: 753-767.

Kimmerer RW. 1993. Disturbance and dominance in Tetraphis pellucida: a model of disturbance frequency and reproductive mode. The Bryologist 96: 73-79.

Kozlowski J. 1992. Optimal allocation of resources to growth and reproduction: implications for age and size at maturity. Trends in Ecology and Evolution 11: 276-280.

Krellwitz EC, Kowallik KV, Manos PS. 2001. Molecular and morphological analyses of Bryopsis (Bryopsidales, Chlorophyta) from the western North Atlantic and Caribbean. Phycologia 40: 330-339. 
Leon-Vargas Y, Engwald S, Proctor MCF. 2006. Microclimate, light adaptation and desiccation tolerance of epiphytic bryophytes in two Venezuelan cloud forests. Journal of Biogeography 33: 901-913.

Löbel S. 2009. Metapopulation and metacommunity processes, dispersal strategies and life-history trade-offs in epiphytes. Uppsala, Sweden: Uppsala University.

Löbel S, Rydin H. 2010. Trade-offs and habitat constraints in the establishment of epiphytic bryophytes. Functional Ecology 24: 887-897.

Löbel S, Snäll T, Rydin H. 2006a. Metapopulation processes in epiphytes inferred from patterns of regional distribution and local abundance in fragmented forest landscapes. Journal of Ecology 94: 856-868.

Löbel S, Snäll T, Rydin H. 2006b. Species richness patterns and metapopulation processes - evidence from epiphyte communities in boreo-nemoral forests. Ecography 29: 169-182.

Löbel S, Snäll T, Rydin H. 2009. Sexual system, reproduction mode and diaspore size affect metacommunity diversity. Journal of Ecology 97: 176-185.

Longton RE. 1997. Reproductive biology and life-history strategies. Advances in Bryology 6: 65-101.

Longton RE, Schuster RM. 1983. Reproductive biology. In: Schuster RM, ed. New manual of bryology. Nichinan: Hattori Botanical Laboratory, 386-462.

Maddison WP, Maddison DR. 1992. MacClade: analysis of phylogeny and character evolution, version 4.0. Sunderland, MA: Sinauer Associates.

McDaniel SF, von Stackelberg M, Richardt S, Quatrano RS, Reski R, Rensing SA. 2010. The speciation history of the PhyscomitriumPhyscomitrella species complex. Evolution 64: 217-231.

Miller JS, Venable DL. 2003. Floral morphometrics and the evolution of sexual dimorphism in Lycium (Solanaceae). Evolution 57: 74-86.

Mishler BD, Newton AE. 1988. Influences of mature plants and desiccation on germination of spores and gametophytic fragments of Tortula. Journal of Bryology 15: 327-342.

Mogie M. 1992. The evolution of sexual reproduction in plants. London: Chapman \& Hall.

Nadot S, Bajon R, Lejeune B. 1994. The choloroplast gene rps4 as a tool for the study of Poaceae phylogeny. Plant Systematics and Evolution 191: 27-38.

Nylander JAA. 2004. MrModeltest v2. Uppsala, Sweden: Evolutionary Biology Centre, Uppsala University.

Pacak A, Szweykowska-Kulinska Z. 2000. Molecular data concerning alloploid character and the origin of chloroplast and mitochondrial genomes in the liverwort species Pellia borealis. Journal of Plant Biotechnology 2: 101-108.

Pagel M. 1994. Detecting correlated evolution on phylogenies: a general method for the comparative analysis of discrete characters. Proceedings of the Royal Society of London. Series B 255: 37-45.

Pagel M. 1999. The maximum likelihood approach to reconstructing ancestral character states of discrete characters on phylogenies. Systematic Biology 48: 612-622.

Pagel M, Lutzoni F. 2002. Accounting for phylogenetic uncertainty in comparative studies of evolution and adaptation. In: Lässig M, Valleriani A, eds. Biological evolution and statistical physics. Berlin: Springer, 148-161.

Pagel M, Meade A, Barker D. 2004. Bayesian estimation of ancestral character states on phylogenies. Systematic Biology 53: 673-684.

Parsons G, Cairns A, Johnson CN, Robson SKA, Shilton LA, Westcott DA. 2007. Bryophyte dispersal by flying foxes: a novel discovery. Oecologia 152: 112-114.

Pohjamo M, Laaka-Lindberg S. 2003. Reproductive modes in a leafy hepatic Anastrophyllum hellerianum. Perspectives in Plant Ecology, Evolution and Systematics 6: 159-168.

Pohjamo M, Laaka-Lindberg S, Ovaskainen O, Korpelainen H. 2006. Dispersal potential of spores and asexual propagules in the epixylic hepatic Anastrophyllum hellerianum. Evolutionary Ecology 20: 415-430.
Proctor MCF, Oliver MJ, Wood AJ, Alpert P, Stark LR, Cleavitt NL, Mishler BD. 2007. Desiccation-tolerance in bryophytes: a review. Bryologist 110: 595-621.

Rambaut A, Drummond AJ. 2007. Tracer v1.4 [WWW document]. URL http://beast.bio.ed.ac.uk/Tracer [accessed 27 May 2011].

Renner SS, Beenken L, Grimm GW, Kocyan A, Rickelfs RE. 2007. The evolution of dioecy, heterodichogamy, and labile sex expression in Acer. Evolution 61: 2701-2719.

Renner SS, Ricklefs RE. 1995. Dioecy and its correlates in the flowering plants. American Journal of Botany 82: 596-606.

Renner SS, Won H. 2001. Repeated evolution of dioecy from monoecy in Siparunaceae (Laurales). Systematic Biology 50: 700-712.

Robert EC. 2004. MUSCLE: multiple sequence alignment with high accuracy and high throughput. Nucleic Acids Research 32: 1792-1797.

Ronquist F, Huelsenbeck JP. 2003. MrBayes 3: Bayesian phylogenetic inference under mixed models. Bioinformatics 19: 1572-1574.

Ronse De Craene LP, Wanntorp L. 2006. Evolution of floral characters in Gunnera (Gunneraceae). Systematic Botany 31: 671-688.

Rydgren K, Cronberg N, Økland RH. 2006. Factors influencing reproductive success in the clonal moss, Hylocomium splendens. Oecologia 147: 445-454.

Rydgren K, Økland R. 2003. Short-term costs of sexual reproduction in the clonal moss Hylocomium splendens. Bryologist 106: 212-220.

Sakai AK, Wagner DL, Ferguson DM, Herbst DR. 1995. Origins of dioecy in the Hawaiian flora. Ecology 76: 2517-2529.

Schuster RM. 1966. The Hepaticae and Anthocerotae of North America east of the hundredth meridian. Volume 1. New York: Columbia University Press.

Smith SD. 2010. Using phylogenetics to detect pollinator-mediated floral evolution. New Phytologist 188: 354-363.

Snäll T, Ehrlen J, Rydin H. 2005. Colonization-extinction dynamics of an epiphyte metapopulation in a dynamic landscape. Ecology 86 : 106-115.

Snäll T, Fogelqvist J, Ribeiro PJ, Lascoux M. 2004a. Spatial genetic structure in two congeneric epiphytes with different dispersal strategies analysed by three different methods. Molecular Ecology 13: 2109-2119.

Snäll T, Hagstrom A, Rudolphi J, Rydin H. 2004b. Distribution pattern of the epiphyte Neckera pennata on three spatial scales - importance of past landscape structure, connectivity and local conditions. Ecography 27: 757-766.

Söderström L, Herben T. 1997. Dynamics of bryophyte metapopulations. Advances in Bryology 6: 205-240.

Soule M. 1971. The variation problem: the gene flow-variation hypothesis. Taxon 20: 37-50.

Souza-Chies TT, Bittar G, Nadot S, Carter L, Besin E, Lejeune B. 1997. Phylogenetic analysis of the Iridaceae with parsimony and distance methods using the plastid gene rps4. Plant Systematics and Evolution 204: 109-123

Stark LR, Brinda JC, McLetchie DN. 2009. An experimental demonstration of the cost of sex and a potential resource limitation on reproduction in the moss Pterygoneurum (Pottiaceae). American Journal of Botany 96: 1712-1721.

Stearns SC. 1989. Trade-offs in life history evolution. Functional Ecology 3: 259-268.

Swofford DL. 2002. PAUP*: Phylogenetic analysis using parsimony (*and other methods), version 4. Sunderland, MA: Sinauer.

Taberlet P, Gielly L, Pautou G, Bouvet J. 1991. Universal primers for amplification of three non-coding regions of chloroplast DNA. Plant Molecular Biology 17: 1105-1109.

Taylor PJ, Eppley SM, Jesson LK. 2007. Sporophytic inbreeding depression in mosses occurs in a species with separate sexes but not in a species with combined sexes. American Journal of Botany 94 : $1853-1859$ 
Vanderpoorten A, Goffinet B. 2009. Introduction to bryophytes. Cambridge: Cambridge University Press.

Vanderpoorten A, Gradstein SR, Carine MA, Devos N. 2010. The ghosts of Gondwana and Laurasia in modern liverwort distributions. Biological Reviews 85: 471-487.

Weller SG, Sakai AK. 1999. Using phylogenetic approaches for the analysis of plant breeding system evolution. Annual Review of Ecology and Systematics 30: 167-199.

Wilson WG, Harder LD. 2003. Reproductive uncertainty and the relative competitiveness of simultaneous hermaphroditism versus dioecy. The American Naturalist 162: 220-241.

Yamada K. 2003. Radulaceae. In: Gradstein SR, Costa DP, eds. The hepaticae and anthocerotae of Brazil. Memoirs of the New York Botanical Garden 87: 1-318.

Zheng M, Zhu RL. 2009. Karyological studies on some species of Radula (Radulaceae, Jungermanniopsida, Marchantiophyta). Nova Hedwigia 88: 229-244.

\section{Supporting Information}

Additional supporting information may be found in the online version of this article.
Table S1 List of taxa sequenced for this study, GenBank accessions numbers for the sequences produced and lifehistory traits scored

Table S2 List of references consulted for the scoring of lifehistory traits in the genus Radula

Please note: Wiley-Blackwell are not responsible for the content or functionality of any supporting information supplied by the authors. Any queries (other than missing material) should be directed to the New Phytologist Central Office.

\section{About New Phytologist}

1.

- New Phytologist is owned by a non-profit-making charitable trust dedicated to the promotion of plant science, facilitating projects from symposia to open access for our Tansley reviews. Complete information is available at www.newphytologist.org.

- Regular papers, Letters, Research reviews, Rapid reports and both Modelling/Theory and Methods papers are encouraged. We are committed to rapid processing, from online submission through to publication 'as-ready' via Early View - our average submission to decision time is just 29 days. Online-only colour is free, and essential print colour costs will be met if necessary. We also provide 25 offprints as well as a PDF for each article.

- For online summaries and ToC alerts, go to the website and click on 'Journal online'. You can take out a personal subscription to the journal for a fraction of the institutional price. Rates start at $£ 149$ in Europe/\$276 in the USA \& Canada for the online edition (click on 'Subscribe' at the website).

- If you have any questions, do get in touch with Central Office (np-centraloffice@lancaster.ac.uk; tel +44 1524 594691) or, for a local contact in North America, the US Office (newphytol@ornl.gov; tel +1 8655765261 ). 